\title{
Can We Skip Intraoperative Evaluation of Sentinel Lymph Nodes? Nomogram Predicting Involvement of Three or More Axillary Lymph Nodes before Breast Cancer Surgery
}

\author{
Soo Kyung Ahn, MD \\ Min Kyoon Kim, MD, PhD² \\ Jongjin Kim, MD ${ }^{3}$ \\ Eunshin Lee, MD ${ }^{3}$ \\ Tae-Kyung Yoo, MD \\ Han-Byoel Lee, MD ${ }^{3}$ \\ Young Joon Kang, $\mathrm{MD}^{3}$ \\ Jisun Kim, MD, $P h D^{5}$ \\ Hyeong-Gon Moon, MD, PhD \\ Jung Min Chang, MD, $\mathrm{PhD}{ }^{6}$ \\ Nariya Cho, MD, $\mathrm{PhD}^{6}$ \\ Woo Kyung Moon, MD, $\mathrm{PhD}^{6}$ \\ In Ae Park, MD, PhD \\ Dong-Young Noh, MD, $\mathrm{PhD}^{3}$ \\ Wonshik Han, MD, $\mathrm{PhD}{ }^{3}$
}

${ }^{1}$ Department of Surgery, Hallym University Kangnam Sacred Heart Hospital, Seoul, ${ }^{2}$ Department of Surgery, Chung-Ang University College of Medicine, Seoul, ${ }^{3}$ Department of Surgery, Seoul National University College of Medicine, Seoul, ${ }^{4}$ Department of Surgery, Seoul St. Mary's Hospital, Seoul, ${ }^{5}$ Department of Surgery, Asan Medical Center, Seoul, Departments of ${ }^{6}$ Radiology and ${ }^{7}$ Pathology, Seoul National University College of Medicine, Seoul, Korea

Correspondence: Wonshik Han, MD, PhD Department of Surgery, Seoul National University College of Medicine, 101 Daehak-ro, Jongno-gu, Seoul 03080, Korea

Tel: 82-2-2072-1958

Fax: 82-2-3673-4250

E-mail: hanw@snu.ac.kr

Received October 4, 2016

Accepted January 17, 2017

Published Online January 25, 2017

*Soo Kyung Ahn and Min Kyoon Kim contributed equally to this work.

\section{Purpose}

The American College of Surgeons Oncology Group Z0011 trial reported that complete dissection of axillary lymph nodes (ALNs) may not be warranted in women with clinical T1-T2 tumors and one or two involved ALNs who were undergoing lumpectomy plus radiation followed by systemic therapy. The present study was conducted to identify preoperative imaging predictors of $\geq 3$ ALNs.

\section{Materials and Methods}

The training set consisted of 1,917 patients with clinical T1-T2 and node negative invasive breast cancer. Factors associated with $\geq 3$ involved ALNs were evaluated by logistic regression analysis. The validation set consisted of 378 independent patients. The nomogram was applied prospectively to 512 patients who met the Z0011 criteria.

\section{Results}

Of the 1,917 patients, 204 (10.6\%) had $\geq 3$ positive nodes. Multivariate analysis showed that involvement of $\geq 3$ nodes was significantly associated with ultrasonographic and chest computed tomography findings of suspicious ALNs ( $p<0.001$ each). These two imaging criteria, plus patient age, were used to develop a nomogram calculating the probability of involvement of $\geq 3 \mathrm{ALNs}$. The areas under the receiver operating characteristic curve of the nomogram were 0.852 (95\% confidence interval [Cl], 0.820 to 0.883$)$ for the training set and $0.896(95 \% \mathrm{Cl}, 0.836$ to 0.957$)$ for the validation set. Prospective application of the nomogram showed that 60 of 512 patients (11.7\%) had scores above the cut-off. Application of the nomogram reduced operation time and cost, with a very low re-operation rate (1.6\%).

\section{Conclusion}

Patients likely to have $\geq 3$ positive ALNs could be identified by preoperative imaging. The nomogram was helpful in selective intraoperative examination of sentinel lymph nodes.

\section{Key words}

Sentinel lymph node, Nomograms, Z0011, Breast neoplasms, Chest, Computed tomography, Axilla sonography 


\section{Introduction}

Major changes have occurred in the standard management of the axilla in patients with invasive breast cancer, with standard treatment progressing from axillary lymph node dissection (ALND) to sentinel lymph node biopsy (SLNB). Intraoperative pathologic evaluation of sentinel lymph nodes (SLNs) has changed surgical practice and has the advantage that it allows patients with positive SLNs to avoid reoperation by immediately proceeding to ALND.

Recently reported results of the American College of Surgeons Oncology Group (ACOSOG) Z0011 trial indicate that complete ALND does not improve survival in women with clinical T1-T2 tumors and one or two involved axillary nodes who are undergoing lumpectomy with radiation therapy followed by systemic therapy [1]. Thus, a significant proportion of these patients may not require ALND, reducing the usefulness of intraoperative pathologic assessment [2]. However, intraoperative evaluation of SLN is highly effective at detecting the subgroup of patients who may benefit from ALND during the same surgical procedure [3].

Advances in preoperative imaging have further changed the algorithm for axillary management. Preoperative knowledge of axillary lymph node (ALN) involvement has improved individualized multidisciplinary treatment options [4]. In this study, we investigated whether preoperative imaging and clinicopathological factors could be used to identify patients with high tumor burden in ALNs, thus allowing the selection of patients requiring intraoperative analysis of SLNs. Based on preoperative imaging and clinicopathological factors, we also sought to develop a nomogram for use in clinical practice that would predict the probability of the involvement of three or more ALNs.

\section{Materials and Methods}

The Seoul National University Hospital Breast Care Center Database (SNUHBCC database) [5] was reviewed to identify patients with clinical T1-T2 invasive breast cancer and clinically negative ALNs who underwent surgery at Seoul National University Hospital (SNUH) between January 2006 and December 2011. Detailed information regarding the SNUHBCC database that was prospectively collected after obtaining institutional review board approval has been reported [5]. For this study, 1,917 consecutive patients who underwent surgery between 2006 and 2010 were considered the training set, whereas the 378 patients who underwent surgery in 2011 were considered the validation set. Patients with a previous history of breast cancer, with palpable ALNs, with in situ cancer on preoperative core biopsy, who received neo-adjuvant chemotherapy, with tumors $>5 \mathrm{~cm}$ on preoperative ultrasonography (US), or with stage IV breast cancer were excluded from both sets. Following validation and development of a nomogram, the latter was applied prospectively to 512 patients with invasive breast cancer who met the selection criteria of the ACOSOG Z0011 trial and were candidates for breast conserving surgery between January 2012 and June 2014.

\section{Preoperative imaging}

US, contrast-enhanced chest computed tomography (CT), and positron emission tomography (PET)-CT were conducted for preoperative staging of the axilla and distant organs. All images were reviewed by specialized radiologists who had been informed that the patients had invasive breast cancer.

Axillary US examination was performed 1 day before surgery. The maximum cortical thickness was measured on a cross-sectional plane perpendicular to the long axis of the lymph node [6]. The probability of lymph node metastasis was classified by US according to the maximum thickness of the cortex and the appearance of fatty hilum. Grades 1-3 were defined as cortical thicknesses of $\leq 1.5 \mathrm{~mm} ;>1.5 \mathrm{~mm}$ but $\leq 2.5 \mathrm{~mm}$; and $>2.5 \mathrm{~mm}$ but $\leq 3.5 \mathrm{~mm}$, respectively; grade 4 as cortical thickness $>3.5 \mathrm{~mm}$ with an intact fatty hilum; and grade 5 as cortical thickness $>3.5 \mathrm{~mm}$ with loss of the fatty hilum.

All included patients underwent preoperative CT using the protocol described for asymptomatic lung and liver metastases [7]. Chest CT in patients with breast cancer was designed to cover the entire liver, the thoracic cavity and both axilla. ALNs were considered tumor-positive if their shortest diameter was $>1 \mathrm{~cm}$, there was loss of fatty hilum, and / or central necrosis was present.

Of the 1,917 patients in the training set, $364(19.1 \%)$ were evaluated by fluorodeoxyglucose (FDG) PET-CT. Standard uptake values were calculated from the amount of FDG injected, body weight, and soft tissue uptake in attenuationcorrected regional images.

\section{Management of ALNs}

The SLNB technique, in which SLNs are detected using a radioisotope technique and/or with a blue dye, has been described previously [8]. Briefly, 1 to 6 hours prior to surgery Tc-99m antimony sulfur colloid $(0.4 \mathrm{mCi})$ was intradermally injected into the quadrant containing the tumor, lymphoscintigraphic images were obtained approximately $40 \mathrm{~min}$ utes later and SLNs were intraoperatively detected using a 
Table 1. Patient characteristics of the training set

\begin{tabular}{|c|c|}
\hline Characteristic & $\begin{array}{c}\text { No. }(\%) \\
(n=1,917)\end{array}$ \\
\hline \multicolumn{2}{|l|}{ Age (yr) } \\
\hline Mean \pm SD & $50.5 \pm 10.2$ \\
\hline Range & $24-85$ \\
\hline \multicolumn{2}{|c|}{ Tumor size by preoperative US (cm) } \\
\hline Mean \pm SD & $2.28 \pm 1.02$ \\
\hline Range & $0.4-5$ \\
\hline \multicolumn{2}{|l|}{ Axillary LN involvement } \\
\hline$\leq 2$ & $1,713(89.4)$ \\
\hline$\geq 3$ & $204(10.6)$ \\
\hline \multicolumn{2}{|l|}{ Axillary US classification } \\
\hline 1 & $657(34.3)$ \\
\hline 2 & $694(36.2)$ \\
\hline 3 & $292(15.2)$ \\
\hline 4 & $159(8.3)$ \\
\hline 5 & $105(5.5)$ \\
\hline Unknown & $10(0.5)$ \\
\hline \multicolumn{2}{|l|}{ Chest CT ALN } \\
\hline Positive & $198(10.3)$ \\
\hline Negative & $1,716(89.5)$ \\
\hline Unknown & $3(0.2)$ \\
\hline \multicolumn{2}{|l|}{ PET-CT ALN } \\
\hline Positive & $105(5.5)$ \\
\hline Negative & $259(13.5)$ \\
\hline Unknown & $1,553(81.0)$ \\
\hline \multicolumn{2}{|l|}{ Surgery-breast } \\
\hline Conservation & $1,368(71.4)$ \\
\hline Mastectomy & $549(28.6)$ \\
\hline \multicolumn{2}{|l|}{ Surgery-axilla } \\
\hline Sentinel LN biopsy only & $1,490(77.7)$ \\
\hline ALND & $427(22.3)$ \\
\hline \multicolumn{2}{|l|}{ Pathology } \\
\hline Ductal & $1,776(92.6)$ \\
\hline Lobular & $71(3.7)$ \\
\hline Other & $70(3.7)$ \\
\hline \multicolumn{2}{|l|}{ Pathologic tumor size $(\mathrm{cm})$} \\
\hline$\leq 2$ & $1,079(56.3)$ \\
\hline$>2$ & $838(43.7)$ \\
\hline \multicolumn{2}{|l|}{ Estrogen receptor } \\
\hline Positive & $1,355(70.7)$ \\
\hline Negative & $542(28.3)$ \\
\hline Unknown & $20(1.0)$ \\
\hline \multicolumn{2}{|l|}{ Progesterone receptor } \\
\hline Positive & $1,116(58.2)$ \\
\hline Negative & $785(40.9)$ \\
\hline Unknown & $16(0.9)$ \\
\hline
\end{tabular}

Table 1. Continued

\begin{tabular}{lr} 
Characteristic & $\begin{array}{c}\text { No. }(\%) \\
(\mathbf{n}=1,917)\end{array}$ \\
HER2 receptor & \\
Positive & $257(13.4)$ \\
Negative & $1,600(83.5)$ \\
Unknown & $60(3.1)$ \\
\hline
\end{tabular}

US, ultrasonography; LN, lymph node; CT, computed tomography; ALN, axillary lymph node; PET, positron emission tomography; ALND, axillary lymph node dissection; HER2, human epidermal growth factor receptor 2.

gamma probe (NEO2000, Neoprobe Co., Dublin, OH). Four areas around the areola were injected intradermally with $0.8 \%$ indigo carmine $(1 \mathrm{~mL})$ dye immediately before surgery. SLNs were identified as any nodes with radioactive counts $\geq 10 \%$ higher than that of the most radioactive node and/or those stained blue. SLNs and grossly enlarged non-SLNs suspicious for metastasis were harvested, with most bisected and examined intraoperatively by hematoxylin and eosin staining of frozen sections. Postoperatively, SLNs were formalin-fixed, paraffin-embedded, and sectioned in $4-\mu \mathrm{m}$ thickness for pathologic examination. Training and validation set patients with positive SLNBs underwent complete level I and level II ALND.

\section{Statistical analysis}

The associations between having $\geq 3$ involved ALNs with patient demographic characteristics, tumor characteristics on preoperative biopsy, and preoperative imaging results were evaluated by Fisher exact tests. Multivariate logistic regression analysis was conducted using a combination of continuous variables (age, tumor size on preoperative US, and ultrasonographic ALN classification) and dichotomized variables (finding suspicious of positive ALN on chest CT). A nomogram predicting the probability of involvement of $\geq 3$ ALNs was then developed based on the multivariate logistic regression model, with a forward stepwise selection method and likelihood-ratio test used to select a subset of all analyzed factors.

The performance of the nomogram was evaluated by receiver operating characteristic (ROC) curve analysis, with calculation of the areas under the ROC curves. The nomogram was calibrated by plotting the observed probability against the predicted probability from the nomogram. A perfectly accurate nomogram prediction model would result in a plot in which the observed and predicted probabilities would fall along a $45^{\circ}$ line. Thus, the distance between the 
pairs and the $45^{\circ}$ line is a measure of the absolute error of prediction of the nomogram [9]. All statistical analyses were performed using SPSS ver. 19.0 (SPSS Inc., Chicago, IL) and the R Software ver. 2.10.0 (http://www.r-project.org/). A $\mathrm{p}<0.05$ was considered statistically significant.

\section{Results}

Patients, tumors, treatments, and preoperative image characteristics are shown in Table 1. The mean age at diagnosis was 50.5 years (range, 24 to 85 years). Of the 1,917 patients in the training set, $204(10.7 \%)$ showed involvement of $\geq 3$ ALNs, and 427 (22.3\%) underwent ALND. The mean number of retrieved SLNs was 3.44 \pm 1.2 (range, 1 to 9).
Chest CT showed findings suspicious of ALN involvement in 198 patients (10.3\%). PET-CT was performed in 364 patients $(19.9 \%)$, among which $105(28.8 \%)$ had findings suspicious of ALN involvement.

\section{Risk factors of having three or more involved nodes}

Univariate logistic regression analysis (Table 2) showed that having three or more involved ALNs was significantly associated with tumor size on preoperative US ( $p<0.001)$, ultrasonographic ALN classification $(\mathrm{p}<0.001)$, and findings suspicious of positive ALN on chest CT $(\mathrm{p}<0.001)$ and PET$\mathrm{CT}(\mathrm{p}<0.001)$, while it was marginally associated with patient age $(\mathrm{p}=0.053)$.

Table 3 shows the sensitivity, specificity, positive predictive value (PPV), and negative predictive value (NPV) of US, $\mathrm{CT}$, and PET-CT in predicting involvement of $\geq 3$ ALNs.

Table 2. Factors associated with involvement of three or more ALNs

\begin{tabular}{|c|c|c|c|}
\hline Variable & Three or more LN (+) (\%) & Two or less LN (+) (\%) & p-value \\
\hline Age (yr) & 49.2 & 50.6 & 0.053 \\
\hline Tumor size by preoperative US (cm) & $2.66 \pm 1.07$ & $2.23 \pm 1.0$ & $<0.001$ \\
\hline Tumor size by MRI (cm) & $2.86 \pm 1.58$ & $2.17 \pm 1.46$ & $<0.001$ \\
\hline \multicolumn{4}{|l|}{ US tumor size $(\mathrm{cm})$} \\
\hline$\leq 2$ & $68(33.3)$ & $851(49.7)$ & $<0.001$ \\
\hline$>2-5$ & $136(66.7)$ & $862(50.3)$ & \\
\hline \multicolumn{4}{|l|}{ Axillary US classification } \\
\hline Gr 1 & $11(5.4)$ & $646(37.7)$ & $<0.001$ \\
\hline Gr 2 & $34(16.7)$ & $660(38.5)$ & \\
\hline Gr 3 & $38(18.6)$ & $254(14.8)$ & \\
\hline Gr 4 & $57(27.9)$ & $102(6.0)$ & \\
\hline Gr 5 & $62(30.4)$ & $43(2.5)$ & \\
\hline \multicolumn{4}{|l|}{ Chest CT ALN } \\
\hline Positive & $106(52.7)$ & $92(5.4)$ & $<0.001$ \\
\hline Negative & $95(47.3)$ & $1,621(94.6)$ & \\
\hline \multicolumn{4}{|l|}{ PET-CT ALN } \\
\hline Positive & $34(79.1)$ & $71(22.1)$ & $<0.001$ \\
\hline Negative & $9(20.9)$ & $250(77.9)$ & \\
\hline \multicolumn{4}{|l|}{ Estrogen receptor } \\
\hline Positive & $134(67.3)$ & $1,221(71.9)$ & 0.177 \\
\hline Negative & $65(32.7)$ & $477(28.1)$ & \\
\hline \multicolumn{4}{|l|}{ Progesterone receptor } \\
\hline Positive & $125(62.2)$ & $991(58.3)$ & 0.289 \\
\hline Negative & $76(37.8)$ & 709 (41.7) & \\
\hline \multicolumn{4}{|l|}{ HER2 receptor } \\
\hline Positive & $34(17.0)$ & $223(13.5)$ & 0.171 \\
\hline Negative & $166(83.0)$ & $1,434(86.5)$ & \\
\hline
\end{tabular}

Values are presented as mean \pm standard deviation or number (\%). ALN, axillary lymph node; LN, lymph node; US, ultrasonography; MRI, magnetic resonance imaging; CT, computed tomography; PET, positron emission tomography; HER2, human epidermal growth factor receptor 2 . 
Table 3. Sensitivity, specificity, PPV, and NPV for each preoperative image-modality when predicting involvement of three or more axillary lymph nodes

\begin{tabular}{lcccc} 
Variable & Sensitivity (\%) & Specificity (\%) & PPV (\%) & NPV (\%) \\
Axillary US & 66.7 & 88.4 & 43.2 & 95.3 \\
Chest CT & 55.2 & 92.8 & 50.8 & 93.9 \\
PET-CT & 70.7 & 74.5 & 27.2 & 94.9 \\
\hline
\end{tabular}

PPV, positive predictive value; NPV, negative predictive value; US, ultrasonography; $\mathrm{CT}$, computed tomography; PET, positron emission tomography.

Table 4. Multivariate logistic regression analysis of factors associated with involvement of three or more ALNs

\begin{tabular}{lccc} 
Variable & Odds ratio & $95 \%$ CI & p-value \\
Age & 0.99 & $0.97-1.00$ & 0.097 \\
Tumor size by preoperative US $(\mathrm{cm})$ & 1.08 & $0.91-1.28$ & 0.392 \\
Axillary US grade & 2.13 & $1.80-2.52$ & $<0.001$ \\
Chest CT ALN positive & 4.78 & $3.07-7.45$ & $<0.001$ \\
\hline
\end{tabular}

ALN, axillary lymph node; $\mathrm{CI}$, confidence interval; US, ultrasonography; $\mathrm{CT}$, computed tomography.

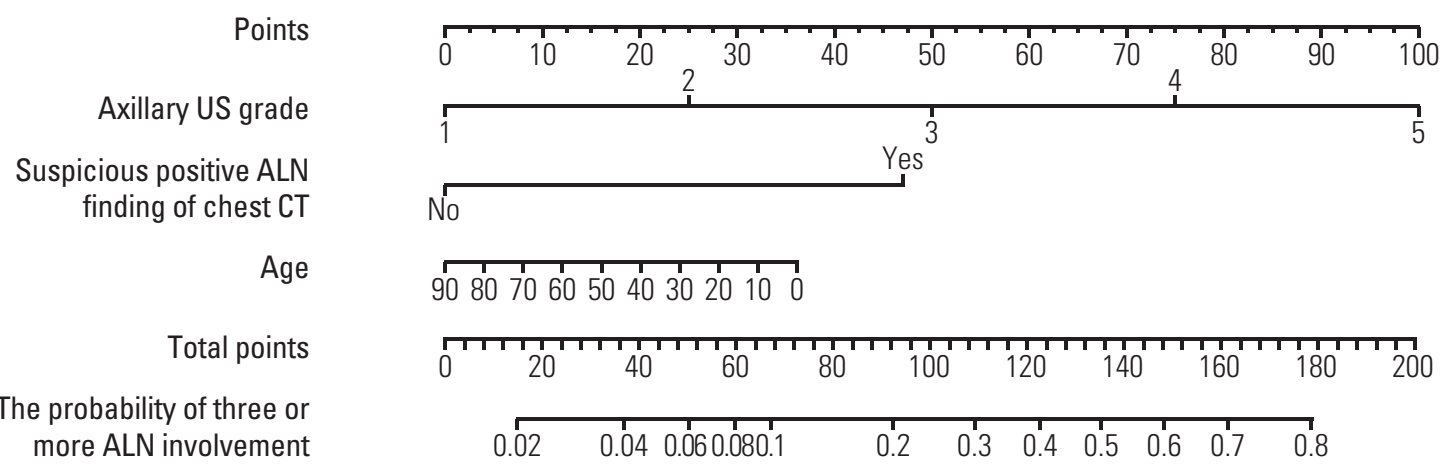

\begin{tabular}{lcccccccccccc}
\hline Total points & 15 & 37 & 50 & 60 & 67 & 92 & 109 & 123 & 135 & 148 & 161 & 178 \\
Likelihood & 0.02 & 0.04 & 0.06 & 0.08 & 0.1 & 0.2 & 0.3 & 0.4 & 0.5 & 0.6 & 0.7 & 0.8 \\
\hline
\end{tabular}

Fig. 1. Nomogram for predicting the probability of having three or more involved axillary lymph node (ALNs). US, ultrasonography; $\mathrm{CT}$, computed tomography.

Multivariate logistic regression analysis was performed using patient age, tumor size on preoperative US (cm), ultrasonographic ALN classification, findings suspicious of positive ALN on chest CT, and findings suspicious of positive ALN on PET-CT. Higher grade ultrasonographic ALN classification (odds ratio [OR], 2.13; 95\% confidence interval [CI],
1.80 to 2.52; $\mathrm{p}<0.001)$ and findings suspicious of positive ALN on chest CT (OR, 4.78; 95\% CI 3.07 to $7.45 ; \mathrm{p}<0.001)$ were found to be independent predictors of having $\geq 3$ involved ALNs. However, findings suspicious of positive ALN on PET-CT were no longer significant $(\mathrm{p}=0.163)$.

Since only $27.3 \%$ of the patients in the training set under- 


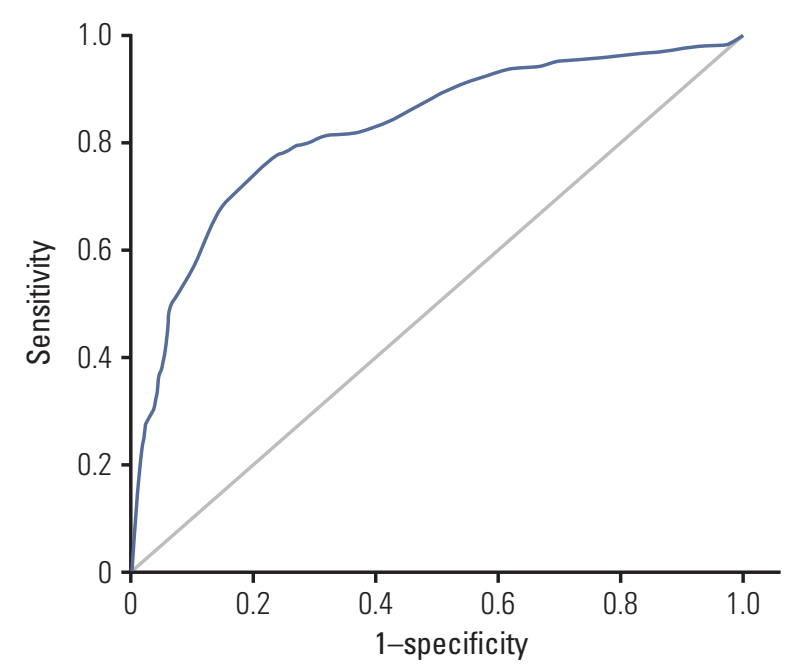

A

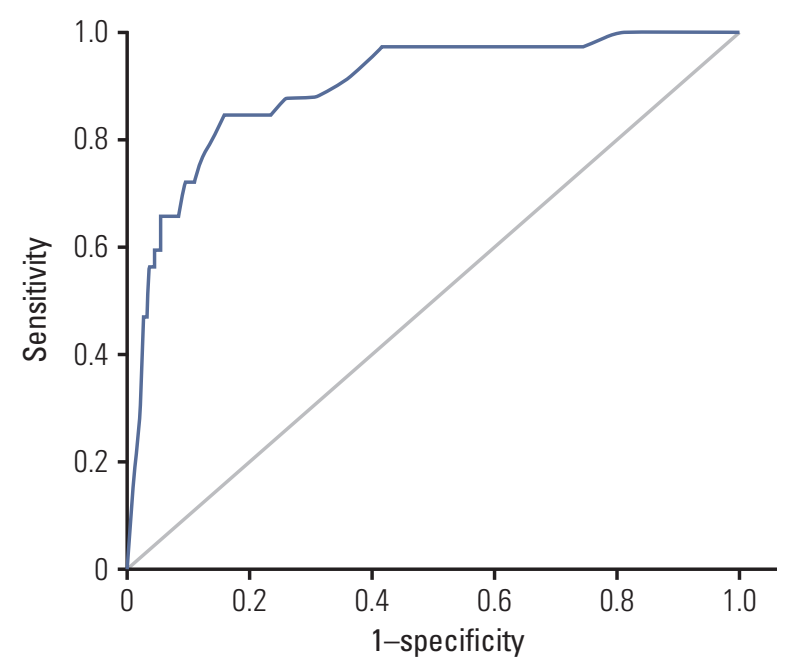

Fig. 2. Performance of the nomogram in the training set and the validation set were each measured using the area under the receiver operating characteristic curves. (A) Training set: 0.852 (95\% confidence level, 0.820 to 0.883 ). (B) Validation set: 0.896 (95\% confidence level, 0.836 to 0.957 ).

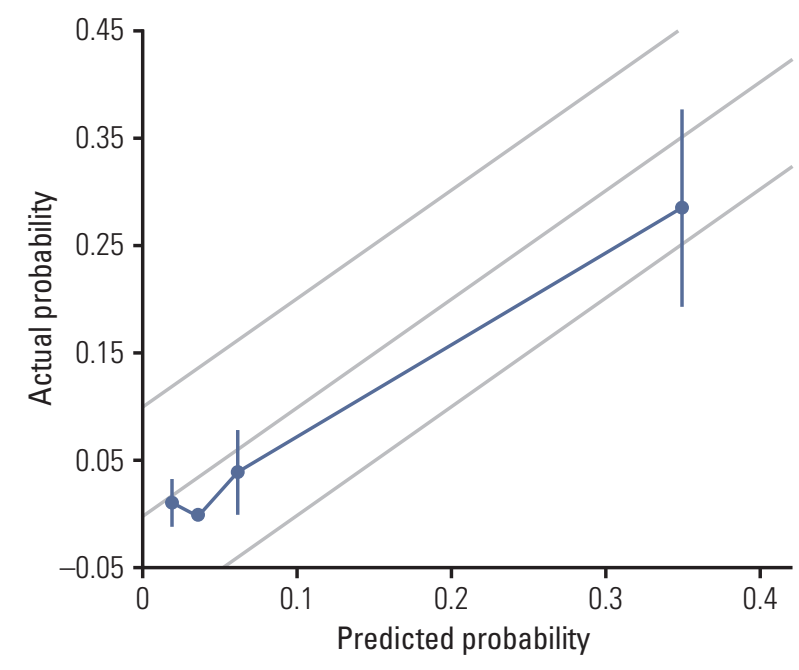

Fig. 3. Calibration plot of the nomogram using validation cohort.

went PET-CT, another multivariate logistic regression analysis was performed excluding the PET-CT findings (Table 4). This analysis showed that higher grade ultrasonographic ALN classification $(\mathrm{p}<0.001)$ and findings suspicious of positive ALN on chest CT $(p<0.001)$ were significantly and independently correlated with an increased probability of having $\geq 3$ involved ALNs.

\section{Nomogram development and validation}

The results of the multivariate analysis were used to develop a nomogram predicting the likelihood of involvement of $\geq 3$ positive ALNs for use in clinical practice. Points were assigned to each variable, such as patient age at diagnosis, axillary US grade, and positive ALN findings on chest $\mathrm{CT}$, then summed to yield the total number of points. The latter was used to assign a probability of $\geq 3$ positive ALNs for individual patients using the scale at the bottom of Fig. 1. The areas under the ROC curves were 0.852 (95\% CI, 0.820 to 0.883 ) for the training set and $0.896(95 \% \mathrm{CI}, 0.836$ to 0.957) for the validation set (Fig. 2).

To assess the accuracy of the nomogram, the actual probability was plotted against the calculated predicted probability of having $\geq 3$ involved ALNs for each patient in the external validation set (Fig. 3). The overall predictive accuracy of the model was within a margin of error of $10 \%$.

The specificity and NPV of the nomogram were calculated and the ability of the nomogram to correctly classify patients into two groups (those with $\leq 2$ or $\geq 3$ involved ALNs) was determined based on whether the predicted probability was more or less than $40 \%$. When using $\leq 123$ points as a cut-off, the specificity of the nomogram was $90 \%$ and its NPV was $95.8 \%$. Of the 1,610 patients in the training set predicted to have $\leq 2$ involved ALNs, only $68(4.2 \%)$ showed false-negative results, resulting in a reoperation rate of ALND $<5 \%$. Thus, this cut-off could be used to define a subset of patients that could avoid intraoperative frozen analysis. Use of the 
same cut-off in the validation set of 378 patients showed that $307(81.2 \%)$ had $\leq 2$ involved ALNs, of which only seven $(2.3 \%)$ showed false-negative results (i.e., $\geq 3$ involved ALNs).

\section{Prospective application of the nomogram}

The nomogram was subsequently applied to a prospective cohort of 512 patients (S1 Table). Of these, 452 patients $(88.3 \%)$ had nomogram scores below the cut-off and could avoid intraoperative frozen analysis of SLNs. Thus, the costs of frozen biopsy and operation time could be reduced (data not shown). Of the patients with a low score and without frozen biopsy, only eight (1.6\% of all 512 patients) had $\geq 3$ involved SLNs and required reoperation for complete ALND (S2 Table). However, without applying the nomogram, 22 patients $(4.3 \%)$ would have undergone reoperation.

\section{Discussion}

The effectiveness of intraoperative evaluation of SLN biopsy remains unclear [10-12]. This method is used to identify patients with metastatic SLNs during the primary surgery, enabling them to avoid a possible second operation. However, the examination rate of intraoperative frozen sections of SLN has been declining [13] for several reasons. First, the yield of the frozen section procedure is generally low, and it is even lower for certain low-risk patients. For example, when used to evaluate women aged $\geq 60$ years with T1a, $\mathrm{b}$ tumors only $3 \%-8 \%$ are found to be positive by frozen biopsies [14]. Additionally, a review of currently available data revealed that the false-negative rate of intraoperative frozen sections of SLN was 14\%-43\% [15-21]. Moreover, patients with positive frozen biopsies of SLN who undergo immediate ALND may require reoperation, many for tumorinvolved resection margins [22]. Finally, the ACOSOG Z0011 trial found that a significant proportion of patients, including women with clinical T1-T2 invasive breast cancer, no palpable adenopathy, one to two sentinel LNs containing metastases, and scheduled for whole breast radiation and systemic therapy, may not require ALND at all.

Predicting ALN status prior to surgery can facilitate surgical planning and informed discussions with patients regarding management options. Various nonsurgical methods including US, CT scan, PET imaging, and magnetic resonance imaging have been tried to predict lymph node involvement [23-29]; however, their predictive accuracies were not high enough to replace SLN biopsy. A previous study conducted by our group revealed that US classification of ALN showed $85 \%$ sensitivity and $78 \%$ specificity for predicting metastasis, with an area under the curve of 0.861 (95\% CI, 0.796 to 0.926 ) [6]. A recent review of 21 studies of preoperative US-guided needle biopsy reported a median sensitivity of $64 \%$ and a median specificity of $82 \%$ [30].

We hypothesized that, although it would be difficult to distinguish involvement of zero and at least one $\mathrm{LN}$, it may be possible to predict high LN tumor burden, which was defined as involvement of $\geq 3$ ALNs. Preoperative imaging was able to predict patients with $\geq 3$ positive ALNs, enabling the avoidance of intraoperative analysis of SLNs in patients with low scores on the nomogram, thereby reducing operation times and costs. Using this method, the rate of reoperation was very low.

Findings suspicious of metastatic ALN on chest CT were a significant predictor of high tumor burden in the axilla, making chest CT results a major component of our nomogram. This is one of the limitations of this study, since chest $\mathrm{CT}$ is not a routinely performed imaging modality for early stage breast cancer patients in most institutions. However, our findings indicate the necessity of performing chest $\mathrm{CT}$ to use our nomogram in practice. In contrast to a small study of 35 breast cancer patients, which reported that chest CT had a low NPV (20\%) for axillary metastases [26], we found a high NPV of $93.9 \%$ and a specificity of $92.8 \%$ for predicting $\geq 3$ metastatic ALNs.

PET-CT has been utilized more frequently than chest CT to evaluate ALN metastasis. In our study, PET-CT had a low PPV (27.2\%) and was not significant in multivariate analysis $(\mathrm{p}=0.163)$. This may have been due to the relatively low percentage $(19.9 \%)$ of our patients evaluated by PET-CT. Further investigations are required to determine whether adding PET-CT to our nomogram would enhance its performance.

Two strategies are deemed feasible for patients who meet the criteria of the ACOSOG Z0011 trial: to never or always evaluate intraoperative frozen biopsy samples. Use of our nomogram could reduce the reoperation rate when compared with the non-use of frozen biopsy, while saving time and costs compared with universal use of frozen biopsy.

It should be noted that this model had several limitations in addition to those associated with the use of chest CT. Specifically, US classification of ALN may be subjective and has not been validated in other institutions.

In summary, we showed that patients with a high probability of having $\geq 3$ positive ALNs can be identified using preoperative imaging methods, such as CT and US, as well as patient demographic and clinical characteristics. The developed nomogram may be useful for identifying patients who do not require intraoperative analysis of SLNs. 


\section{Electronic Supplementary Material}

Supplementary materials are available at Cancer Research and Treatment website (http:// www.e-crt.org).

\section{Conflicts of Interest}

Conflict of interest relevant to this article was not reported.

\section{Acknowledgments}

This study was supported by grant of the National Research Foundation of Korea (NRF) grant funded by the Korea government (MSIP) (2015R1A2A2A01008264).

\section{References}

1. Giuliano AE, Hunt KK, Ballman KV, Beitsch PD, Whitworth PW, Blumencranz PW, et al. Axillary dissection vs no axillary dissection in women with invasive breast cancer and sentinel node metastasis: a randomized clinical trial. JAMA. 2011;305: 569-75.

2. Setton J, Cody H, Tan L, Morrow M, Hudis C, Catalano J, et al. Radiation field design and regional control in sentinel lymph node-positive breast cancer patients with omission of axillary dissection. Cancer. 2012;118:1994-2003.

3. Taffurelli M, Montroni I, Santini D, Fiacchi M, Zanotti S, Ugolini G, et al. Effectiveness of sentinel lymph node intraoperative examination in 753 women with breast cancer: are we overtreating patients? Ann Surg. 2012;255:976-80.

4. Valente SA, Levine GM, Silverstein MJ, Rayhanabad JA, Weng-Grumley JG, Ji L, et al. Accuracy of predicting axillary lymph node positivity by physical examination, mammography, ultrasonography, and magnetic resonance imaging. Ann Surg Oncol. 2012;19:1825-30.

5. Moon HG, Han W, Noh DY. Underweight and breast cancer recurrence and death: a report from the Korean Breast Cancer Society. J Clin Oncol. 2009;27:5899-905.

6. Cho N, Moon WK, Han W, Park IA, Cho J, Noh DY. Preoperative sonographic classification of axillary lymph nodes in patients with breast cancer: node-to-node correlation with surgical histology and sentinel node biopsy results. AJR Am J Roentgenol. 2009;193:1731-7.

7. Kim H, Han W, Moon HG, Min J, Ahn SK, Kim TY, et al. The value of preoperative staging chest computed tomography to detect asymptomatic lung and liver metastasis in patients with primary breast carcinoma. Breast Cancer Res Treat. 2011;126: 637-41.

8. Han A, Moon HG, Kim J, Ahn SK, Park IA, Han W, et al. Reliability of sentinel lymph node biopsy after neoadjuvant chemotherapy in breast cancer patients. J Breast Cancer. 2013; 16:378-85.

9. Iasonos A, Schrag D, Raj GV, Panageas KS. How to build and interpret a nomogram for cancer prognosis. J Clin Oncol. 2008;26:1364-70.

10. Cipolla C, Cabibi D, Fricano S, Vieni S, Gentile I, Latteri MA. The value of intraoperative frozen section examination of sentinel lymph nodes in surgical management of breast carci- noma. Langenbecks Arch Surg. 2010;395:685-91.

11. Schwartz GF, Krill LS, Palazzo JP, Dasgupta A. Value of intraoperative examination of axillary sentinel nodes in carcinoma of the breast. J Am Coll Surg. 2008;207:758-62.

12. Liu W, Zakharia Y, Tan W, Wilding G, Edge S, Tan D, et al. Intraoperative detection of sentinel lymph node metastases from breast cancer: does the pathologist experience, subspecialty or the surgeon experience matter? Lab Invest. 2010;90: 59A.

13. Weber WP, Barry M, Stempel MM, Junqueira MJ, Eaton AA, Patil SM, et al. A 10-year trend analysis of sentinel lymph node frozen section and completion axillary dissection for breast cancer: are these procedures becoming obsolete? Ann Surg Oncol. 2012;19:225-32.

14. Chan SW, LaVigne KA, Port ER, Fey JV, Brogi E, Borgen PI, et al. Does the benefit of sentinel node frozen section vary between patients with invasive duct, invasive lobular, and favorable histologic subtypes of breast cancer? Ann Surg. 2008;247:143-9.

15. Chao C, Wong SL, Ackermann D, Simpson D, Carter MB, Brown CM, et al. Utility of intraoperative frozen section analysis of sentinel lymph nodes in breast cancer. Am J Surg. 2001; 182:609-15.

16. Tanis PJ, Boom RP, Koops HS, Faneyte IF, Peterse JL, Nieweg $\mathrm{OE}$, et al. Frozen section investigation of the sentinel node in malignant melanoma and breast cancer. Ann Surg Oncol. 2001;8:222-6.

17. Weiser MR, Montgomery LL, Susnik B, Tan LK, Borgen PI, Cody HS. Is routine intraoperative frozen-section examination of sentinel lymph nodes in breast cancer worthwhile? Ann Surg Oncol. 2000;7:651-5.

18. Rahusen FD, Pijpers R, Van Diest PJ, Bleichrodt RP, Torrenga $\mathrm{H}$, Meijer S. The implementation of the sentinel node biopsy as a routine procedure for patients with breast cancer. Surgery. 2000;128:6-12.

19. Veronesi U, Paganelli G, Viale G, Galimberti V, Luini A, Zurrida $S$, et al. Sentinel lymph node biopsy and axillary dissection in breast cancer: results in a large series. J Natl Cancer Inst. 1999;91:368-73.

20. Noguchi M, Bando E, Tsugawa K, Miwa K, Yokoyama K, Nakajima K, et al. Staging efficacy of breast cancer with sen- 
tinel lymphadenectomy. Breast Cancer Res Treat. 1999;57: 221-9.

21. Canavese G, Gipponi M, Catturich A, Di Somma C, Vecchio $\mathrm{C}$, Rosato F, et al. Sentinel lymph node mapping opens a new perspective in the surgical management of early-stage breast cancer: a combined approach with vital blue dye lymphatic mapping and radioguided surgery. Semin Surg Oncol. 1998; 15:272-7.

22. McLaughlin SA, Ochoa-Frongia LM, Patil SM, Cody HS 3rd, Sclafani LM. Influence of frozen-section analysis of sentinel lymph node and lumpectomy margin status on reoperation rates in patients undergoing breast-conservation therapy. J Am Coll Surg. 2008;206:76-82.

23. Pamilo M, Soiva M, Lavast EM. Real-time ultrasound, axillary mammography, and clinical examination in the detection of axillary lymph node metastases in breast cancer patients. J Ultrasound Med. 1989;8:115-20.

24. Nori J, Vanzi E, Bazzocchi M, Bufalini FN, Distante V, Branconi $\mathrm{F}$, et al. Role of axillary ultrasound examination in the selection of breast cancer patients for sentinel node biopsy. Am J Surg. 2007;193:16-20.
25. Lee MC, Eatrides J, Chau A, Han G, Kiluk JV, Khakpour N, et al. Consequences of axillary ultrasound in patients with $\mathrm{T} 2$ or greater invasive breast cancers. Ann Surg Oncol. 2011;18:72-7.

26. March DE, Wechsler RJ, Kurtz AB, Rosenberg AL, Needleman L. CT-pathologic correlation of axillary lymph nodes in breast carcinoma. J Comput Assist Tomogr. 1991;15:440-4.

27. Peare R, Staff RT, Heys SD. The use of FDG-PET in assessing axillary lymph node status in breast cancer: a systematic review and meta-analysis of the literature. Breast Cancer Res Treat. 2010;123:281-90.

28. Kvistad KA, Rydland J, Smethurst HB, Lundgren S, Fjosne HE, Haraldseth O. Axillary lymph node metastases in breast cancer: preoperative detection with dynamic contrast-enhanced MRI. Eur Radiol. 2000;10:1464-71.

29. Yoshimura G, Sakurai T, Oura S, Suzuma T, Tamaki T, Umemura $\mathrm{T}$, et al. Evaluation of axillary lymph node status in breast cancer with MRI. Breast Cancer. 1999;6:249-58.

30. Houssami N, Ciatto S, Turner RM, Cody HS 3rd, Macaskill P. Preoperative ultrasound-guided needle biopsy of axillary nodes in invasive breast cancer: meta-analysis of its accuracy and utility in staging the axilla. Ann Surg. 2011;254:243-51. 